\title{
PHYSIOLOGICAL CHARACTERISTICS OF DYSPHAGIA FOLLOWING THERMAL BURN INJURY ${ }^{1}$
}

Anna F. Rumbach BSc, MSpPathSt, GCHEd (Corresponding Author/Re-Print requests) School of Health and Rehabilitation Sciences, Division of Speech Pathology, The University of Queensland, St Lucia, Brisbane, 4072, Australia Phone: +61 407123 879; E-mail: a.rumbach@uq.edu.au

Elizabeth C. Ward BSpThy (Hons), Grad Cert Ed., PhD Centre for Functioning and Health Research, Queensland Health Level 3, Centro Buranda, Ipswich Road, Buranda, QLD 4102, Australia; School of Health and Rehabilitation Sciences, Division of Speech Pathology, The University of Queensland, St Lucia, Brisbane, 4072, Australia

\section{Petrea L. Cornwell BSpPath (Hons), PhD}

Metro North Health Service District, Queensland Health, Queensland, Australia; Griffith Health Institute, Behavioural Basis of Health Program, Griffith University, Mt Gravatt, QLD 4122, Australia

\section{Lynell V. Bassett BSpThy}

Speech Pathology Department, Level 2 James Mayne Building, Royal Brisbane \& Women's Hospital, Butterfield Street, Herston, QLD 4029, Australia

\section{Michael J. Muller MBBS MMed Sci FRACS}

Professor Stuart Pegg Adult Burns Centre, Level 4 James Mayne Building, Royal Brisbane \& Women's Hospital, Butterfield Street, Herston, QLD 4029, Australia; Burns,

Trauma \& Critical Care Research Centre, The University of Queensland, Brisbane, Australia

${ }^{1}$ This project was supported by funding from the Royal Brisbane and Women's Hospital (RBWH) Foundation. 
Physiological characteristics of dysphagia following thermal burn injury 


\begin{abstract}
The study aim was to document the acute physiological characteristics of the swallowing impairment following thermal burn injury. A series of 19 participants admitted to a specialised burn centre with thermal burn injury were identified with suspected aspiration risk by a clinical swallow examination (CSE) conducted by a speech-language pathologist and referred to the study. Once medically stable, each then underwent more detailed assessment using both a CSE and a fiberoptic evaluation of swallowing (FEES). Subsequent to this assessment, FEES confirmed 6 individuals (32\%) had no aspiration risk, and were excluded from further analyses. Of the remaining 13, CSE confirmed two had specific oral phase deficits due to orofacial scarring and contractures, and all 13 had generalised oromotor weakness. FEES revealed numerous pharyngeal phase deficits with major finding evident in greater than $50 \%$ being: impaired secretion management, laryngotracheal edema, delayed swallow initiation, impaired sensation, inadequate movement of structures within the hypopharynx and larynx, and diffuse pharyngeal residue. Penetration and/or aspiration occurred in $83 \%(n=10 / 12)$ of thin fluids trials, with a lack of response to the penetration/aspiration noted in $60 \%(\mathrm{n}=$ 6/10 penetration/aspiration events) of cases. Most events occurred post swallow. Findings support that individuals with dysphagia post thermal burn present with multiple risk factors for aspiration that appear predominantly related to generalised weakness and inefficiency and further impacted by edema and sensory impairments. Generalised oromotor weakness and orofacial contractures (when present) impact oral stage swallow function. This study has identified a range of factors which may be contributing to both oral and pharyngeal stage dysfunction in this clinical population and highlights the
\end{abstract}


importance of using a combination of clinical and instrumental assessment to fully understand the influence of burn injury on oral intake and swallowing.

Key Words Deglutition; deglutition disorders; Fiberoptic endoscopic evaluation of swallowing; acute; dysphagia; burn injury; swallowing 


\section{Introduction}

Dysphagia is present in approximately $11 \%$ of individuals who are admitted to hospital for treatment of thermal burn injury ${ }^{1}$ and aspiration risk, as well as the inability to efficiently manage solid food textures, may persist for weeks to months post injury ${ }^{2-7}$. However, despite the clinical significance of dysphagia in this population, to date there has been minimal systematic investigation of the physiological basis for the presenting swallowing impairment. As optimal treatment planning for dysphagia relies on a comprehensive understanding of the physiological deficits that lead to aspiration or aspiration risk, it is important that the numerous factors contributing to dysphagia in this population are documented.

Burns to orofacial regions can result in oral phase difficulties, causing restrictions in range of motion (ROM) that impair lip and cheek movement, as well as jaw opening, thus limiting the proficiency of the oral phase of the swallow ${ }^{4,5-8-12}$. In the presence of severe burns that result in orofacial scarring and contractures, oral deficits may be a longterm limiting factor in returning to normal oral intake ${ }^{4,5}$. These deficits can manifest as poor ability to contain, manipulate and form a cohesive bolus of appropriate size and consistency for swallowing. Whilst the oral phase deficits have largely been discussed in single case reports that utilise clinical swallow examination $(\mathrm{CSE})^{5,6,8,9,12}$, one study which detailed oral phase difficulties also observed during a modified barium swallow (MBS) study noted poor lip closure, decreased ability for mastication and decreased tongue to palate contact ${ }^{4}$. 
While some data are available regarding the nature of oral stage deficits in this population, the specific nature of pharyngeal phase deficits observed after burn injury have been largely undocumented. Muehlberger and colleagues ${ }^{13}$ were the first to detail the physiological characteristics of dysphagia in patients with inhalation injury. Using a MBS assessment with 11 patients who presented clinically with mild dysphagic symptoms, four individuals were described as presenting with mild pharyngeal pooling and delayed swallow initiation. No further discussion of potential factors contributing to the dysphagia in the other 7 cases was discussed. A decade later, Edelman, SheehyDeardorff and White ${ }^{2}$ conducted a retrospective study of 11 burn patients and reported that $64 \%$ presented with oral stage swallowing deficits, $82 \%$ with pharyngeal deficits and $18 \%$ with esophageal dysphagia. Although the authors stated that pharyngeal stage dysphagia was predominantly caused by burn scar formation or inhalation injury no further specific detail of the physiological nature of the deficits were reported ${ }^{2}$.

Currently there is only one case study available that has provided more detailed information about the specific nature of impaired swallow physiology in the burn population. Rumbach et $\mathrm{al}^{4}$ reported on the impact of severe burn injury using fiberoptic endoscopic evaluation of swallowing (FEES) in a 60 year old male. Findings included delayed swallow initiation, decreased laryngopharyngeal sensation, decreased base of tongue (BOT) to posterior pharyngeal wall (PPW) contact as well as reduced superior and anterior hyolaryngeal movement as evidenced by reduced epiglottic deflection and cricopharyngeal opening. Furthermore, supraglottic edema and reduced vocal fold mobility were also observed. However, as this data is limited to a single case of severe 
dysphagia post burn it is uncertain how closely this information may be extrapolated to the wider clinical population with burn injury.

Due to the limited information that currently exists, the aim of the current study is to investigate and describe the acute characteristics of swallowing dysfunction in an adult cohort post thermal burn injury with dysphagia by means of a clinical and instrumental examination. By sampling from a prospective clinical cohort across a range of injury severities, it is intended that the current data will provide a more representative view of the range of swallowing deficits which may be present in this population. Ultimately this data will provide clinicians with detailed information regarding dysphagia characteristics in the thermal burn population to help inform assessment, treatment and rehabilitation planning.

\section{Methods}

\section{Participant Population}

Participants included 19 adults (14 males, 5 females), ranging in age from 18 to 85 years $(M=47.95, S D=20.81)$ with thermal burn injury, with or without inhalation injury, who presented for management at the Royal Brisbane and Women's Hospital specialised burns unit, Australia, over a 24 month period (August 2007- July 2009). The mean total body surface area (TBSA) affected was $31.87 \%(\mathrm{SD}=17.06$, range $=4-66.5)$, with 58\% ( $\mathrm{n}=11)$ having concomitant inhalation injury and 74\% $(\mathrm{n}=14)$ having burns to the head and neck. Over $85 \%$ of participants required periods of mechanical ventilation, with six participants requiring a tracheostomy during the course of their 
hospital admission. No participant had existing neurological or structural impairment that could influence swallowing behaviour or a prior history of swallowing disorders, as determined by medical chart review, multidisciplinary team discussion, and patient report. Biographical data including burn aetiology, severity, subsequent medical management and dysphagia severity prior to and post FEES are detailed for each participant in table 1.

/insert Table 1 near here/

Participants in this cohort diagnosed with orofacial burns $(n=14)$ had received multidisciplinary and interdisciplinary management for contracture prevention from the point of hospital admission as per Rumbach et $\mathrm{al}^{11}$. In addition, prior to the current investigation, all participants had undergone at least one prior CSE by an experienced speech pathologist. The initial referral to speech language pathology (SLP) for dysphagia assessment had occurred on average 16 days post admission $(\mathrm{SD}=15$ days, range $=1-45$ days) once the medical officer in charge had determined the patient to be medically stable and suitable for oral intake. Intervention for suspected aspiration risk as determined from these initial CSEs was limited to compensatory management via texture modifications as participants were deemed not medically stable to participate in active rehabilitation ${ }^{11}$. Referral to the current study occurred only once there was agreement by the multidisciplinary team (including but not limited to the medical officer, speech pathologist, occupational therapist, physiotherapist, psychologist, and dietician) that patients were suitable to undergo an instrumental examination of swallowing to assess aspiration risk and facilitate active rehabilitation planning. Instrumental assessment therefore occurred at various stages of recovery for each patient, at a mean of 37 days 
$(\mathrm{SD}=22$ days, range $=5-80$ days $)$ post injury, and at a mean of 21 days $(\mathrm{SD}=14$ days, range $=4-53$ days) post initial assessment by SLP. At the time of participation in this study, four individuals were tracheostomised, with the cuff deflated and a one-way speaking valve in situ. An additional two individuals had been decannulated 48 hours prior to assessment.

\section{Procedure}

Prior to the study, reliability training in clinical decision making (i.e., dysphagia absence/presence and optimal oral intake) following a CSE was undertaken by the primary rating clinician that involved completing 40 simultaneous CSEs with a second experienced clinician. Percent exact agreement for clinical decisions regarding recommendations for (1) optimal food textures to minimise aspiration risk and (2) fluid consistency to minimise aspiration risk for each patient was $100 \%$ between the two clinicians.

Once deemed suitable for FEES and recruited into this study, all participants underwent a CSE performed by an experienced speech-language pathologist, conducted no more than one day prior to a FEES assessment. This enabled information on both oral and pharyngeal stage deficits to be compiled. The CSE consisted of a patient interview, visual examination of the oromusculature, oromotor examination, perceptual evaluation of voice quality, and a series of oral intake trials of fluids and foods that also included a water swallow test ${ }^{14-17}$ when appropriate. All participants were trialled with the fluids/foods considered to be least normal first (i.e., extremely thick fluids, puree diet), with progression towards normal dietary consistencies and textures (i.e., thin fluids, 
general diet), during oral intake trials if appropriate. Considerations for conducting a CSE with burned individuals, as outlined by Rumbach et $\mathrm{al}^{11}$ were followed, with each assessment requiring some variation depending on patient presentation. From this, an initial rating of dysphagia severity was conducted using a purpose-built rating scale ${ }^{6}$ that ranged from $1=$ normal swallow status (normal diet) to $4=$ severe dysphagia (nil by mouth; small amounts only of oral intake of full texture and consistency restriction). A purpose built severity scale is necessary for this clinical population as most existing dysphagia severity scales factor the need for alternative or supplemental feeding into the severity rating. However, supplemental feeding in the burns population is often not related to dysphagia but rather management of the hypermetabolic response to burn injury, hence most published severity scales cannot be applied to this clinical population.

The FEES procedure was then conducted at the bedside as per protocol ${ }^{18}$ under the direction of an otorhinolaryngologist (ENT) and treating speech pathologist. All images were viewed online using an Olympus Viser OTV-37 digital processor scope with an Olympus Visera CLV-S40 light source attached to a dysphagia swallow workstation (DSW; Kay Pentax). The images were recorded using the DSW, with audio captured by a lapel microphone attached to the patient's collar. Participants were seated upright, and the scope, was passed through the nostril in order to view the nasopharynx, hypopharynx and larynx during non swallow and swallow tasks. Passing of the scope was undertaken by an ENT and no local anaesthesia was used during the procedure.

The initial stage of the FEES involved examination of the structural integrity, symmetry, range, speed and precision/timing/coordination of the velum, BOT, pharyngeal muscles and larynx were assessed through observation of the structures at 
rest, during phonation and a dry swallow. Following this, the observable features of the swallow were documented with the presentation of boluses of different food and fluid textures and consistencies as appropriate for each patient. Dietary consistencies trialled were consistent with the Australian standards for texture modified food and fluids ${ }^{19}$ and the range included smooth puree, minced and moist, soft and normal food consistencies as well as extremely thick (level 900), moderately thick (level 400), mildly thick (level 150) and thin (regular) fluids. All food and fluid was dyed green to allow for easy visualisation of the bolus path. Consistent with the CSE procedure, all participants were trialled with the fluids/foods considered to be least normal first (i.e., extremely thick fluids, puree diet), with progression towards normal dietary consistencies and textures (i.e., thin fluids, general diet), during oral intake trials under FEES. Fluid trials preceded food trials and each participant received at least two trials of each consistency presented. Larger bolus volumes of food and fluid of approximately $20 \mathrm{mls}^{20}$ were used in this study in comparison to volumes typically used in research $\left(5-10 \mathrm{ml}\right.$ boluses $\left.{ }^{21-24}\right)$ to better evaluate aspiration risk with more typical meal bolus sizes. Liquids were presented using a spoon, straw or cup, depending on the patient's ability to self-feed. The participant was instructed to take one sip at a time. Continuous drinking was also assessed on thin fluids if appropriate. Foods were presented via spoon, or as a whole entity (e.g., marshmallow/biscuit) and the participant was instructed to take a normal size bite. Suitability for progression to the next food or fluid texture/consistency was based on (a) the safety of food/fluid intake and (b) the efficiency of fluid/food intake. Compensatory strategies were trialled where appropriate. However, swallowing ability without the use of compensatory measures (other than texture modification) was used in all subsequent 
analysis. All trials of any consistency were ceased in the presence of frank and/or large amounts of penetration/aspiration or if the speech pathologist noted severe swallowing dysfunction with a high risk of aspiration (i.e., not safe for oral intake).

Analysis of the FEES procedure was completed by examination of the video study for each participant at regular speed, slow motion and frame-by-frame. Independent ratings were obtained by two qualified speech pathologists experienced in the area of adult dysphagia and the burns population, including the analysis of the FEES procedure. Rater 1 was present during the online assessment and saw the participants clinically while rater 2 was blinded to the case information for each participant. To evaluate reliability, kappa statistics were calculated for each parameter investigated (Table 2). The results obtained from these analyses were interpreted using strength of agreement ranges as determined by Landis and $\mathrm{Koch}^{25}$. These benchmarks judge reliability coefficient according to the following criteria: $>0.20=$ poor, $0.21-0.40=$ fair, $0.41-0.60=$ moderate, $0.61-0.80=$ good, and $0.81-1.00=$ very good. The kappa values calculated for interrater reliability ranged from 0.7799 to 0.9713 , indicating that good to very good agreement was reached between raters on all parameters evaluated, with the exception of erythema which had fair reliability $(\mathrm{k}=0.24)$. Consensus ratings (as presented in the final data set) were obtained on any item of disagreement among the clinicians. Five of the 19 examinations were chosen and blinded to the two raters, and were re-rated one-month post completion of the original ratings to assess intra-rater reliability (Table 3). Moderate to very good agreement $(\mathrm{k}=0.54-1.00)$ was reached between rating occasions for Rater 1. Rater 2 showed moderate to good agreement $(\mathrm{k}=0.5-0.8)$ between rating occasions. 
/insert Tables 2 and 3 near here/

The analysis parameters used in this study were based on Langmore's ${ }^{26}$ original rating form. Ratings were made of the structural integrity and physiological function of the velum, tongue, pharynx and larynx (as appropriate). The presence of any laryngotracheal pathology (including edema and erythema) was noted. Any edema or erythema of tissues affecting the structural integrity of the hypopharynx and larynx were rated using a purpose-built scale that ranged from 1 (no edema/erythema) to 3 (all tissues edematous/widespread erythema). Adequacy of saliva management was then rated according to the Marionjoy Secretion Rating Scale ${ }^{27}$. This is a 5-level rating scale with ratings ranging from normal (thin, clear secretions with less than $10 \%$ pooling in pyriform fossae or valleculae) to profound (secretions present on vocal folds). Appearance of secretions (colour and viscosity), patient response to secretions (i.e., are attempts to spontaneously clear secretions being made), affect of spontaneous or cued swallows on secretion reduction, and the frequency of spontaneous swallows were also noted to determine adequacy of saliva management prior to the commencement of oral intake trials. Swallow frequency was classified as reduced if spontaneous swallows were observed to be less than 1 per minute. Sensation testing was informally conducted via patient response to the presence and light touch of the endoscope on the lateral pharyngeal walls and tip of the epiglottis. The patient was deemed to have reduced sensation if there was no response to the presence and light taps of the scope in the pharynx and an absent or reduced response to residue or to an event of silent aspiration. The presence of the laryngeal adductor reflex (i.e., brief closure of the true vocal cords), 
an involuntary airway protection reflex, was also examined by light touch of the scope to the arytenoid epithelium.

For the food and fluid trials, each bolus for each consistency trialled was individually rated. Any parameter observed during at least one swallow trial per consistency denoted the characteristic as present for that consistency. Parameters for analysis included observable characteristics of the oral and pharyngeal stage, oral and pharyngeal transit times and other markers indicating poor coordination or inefficiency of the swallow. Specifically, the oral phase of the swallow trials was examined through visual inspection of the acceptance, containment and manipulation of the bolus, oral transit time and an informal rating of delivery of the bolus into the pharynx on a 4-point scale (see appendix A). Ratings ranged from normal (prompt delivery of bolus) to severe impairment (large amount of leakage during oral preparation with minimal mastication of bolus and no attempt at a transfer). At the completion of each bolus, the oral cavity was examined for the presence of oral residue and was rated as being absent or present, with location of any residue being noted.

The pharyngeal phase was examined through determining the adequacy of structural movements during the swallow as per Langmore ${ }^{26}$. This included investigation of bolus driving and clearing forces (i.e., BOT movement, pharyngeal longitudinal and constrictor movement), and valving forces (i.e., velopharyngeal and laryngeal valves). Observations regarding presence of residue after and between swallows were also recorded including information on: location of residue, amount of residue, patient 
awareness of residue, effect of spontaneous or cued swallows on reduction of residue, and number of spontaneous clearing swallows after the patient's first swallow. Informal ratings on reflex initiation, and pharyngeal residue post swallow were also made (see appendix A). Reflex initiation was informally rated on a 4-point scale, which included ratings from normal (bolus dwelled at base of tongue and/or valleculae for 0-1 seconds) to severe impairment (absent reflex). Pharyngeal residue post swallow was also informally rated on a 4-point scale, which consisted of ratings ranging from normal (no residue) to severe impairment (residue filled and/or overflowed cavities). Presence of penetration and/or aspiration before or after the swallow for each consistency trialled was rated on the 8-point Penetration-Aspiration Scale ${ }^{28}$. The ratings ranged from 1 (material did not enter the airway) to 8 (material entered the airway, passed below the vocal folds, and no effort was made to eject it).

From the FEES assessment, safe food and fluid consistencies were determined to be those with which the patient demonstrated no signs of penetration/aspiration, no deficit with efficiency for oral intake or no discomfort. The efficiency of oral intake was determined by the amount of external facilitation/prompting required and/or the amount of oral motor labour demonstrated by the patient in consuming the various food/fluid presented. Based on the outcome of the FEES assessment, dysphagia severity, using the purpose built scale was re-rated and compared to the ratings made following the CSE. 


\section{Results}

At the time of referral for FEES assessment, CSE classified 52.5\% (10/19) of the cohort as having severe dysphagia, 31.5\% (6/19) had a moderate impairment, and $16 \%$ (3/19) had a mild impairment (Table 1). However, subsequently FEES assessment revealed normal swallow function in 6 individuals (participants 2, 3, 4, 6, 13, and 17 Table 1), and these participants were excluded from all subsequent data analysis. Data from this point is represented as a portion of the total remaining cohort of 13 individuals, unless specifically stated otherwise.

Oromotor examination revealed that $77 \%$ of the remaining cohort of 13 presented with a weak or absent voluntary cough and $100 \%$ a dysphonic vocal quality. Poor dentition was also prevalent, with $54 \%$ of the cohort having absent teeth and/or teeth with advanced decay. Decreased lip strength and ROM were evident in $100 \%$ of cases. Jaw strength was also reduced in $54 \%$ of individuals. Nil deficits in tongue function were observed in any participant. Two individuals presented with severe orofacial scarring and contracture formation and presented with severely reduced and asymmetrical orofacial ROM (Table 1). During swallow trials, this degree of scarring and contracture formation caused inadequate labial closure, increased time for oral preparation/bolus formation and diffuse oral residue that required manual removal from the lateral sulci by the clinician for these 2 individuals. No other participant had difficulties controlling oral boluses, yet increased oral preparation time was required for the majority of individuals' trialled on more solid textures (e.g., biscuit). Tightness at the oral commissures, alongside poor dentition, advanced age and reduced jaw strength, lead to fatigue and discomfort in one 
individual; thus limiting their ability to be upgraded to a normal and varied texture diet although aspiration risk has resolved.

FEES assessment of the structural integrity and function of the velopharynx, hypopharynx and larynx prior to the commencement of food and fluid trials are displayed in Table 4. Laryngotracheal pathology, as confirmed by an otorhinolaryngologist, was highly prevalent across the cohort, with $77 \%(\mathrm{n}=10)$ displaying edema, with approximately one third of the cohort exhibiting concurrent tissue granulation, ulceration and/or edema (Table 4). Laryngeal dysfunction was also prevalent in the cohort, with up to $69 \%(n=9)$ of the population presenting with one or more difficulties contributing to altered vocal quality (Table 4). No participants presented with visible nasopharyngeal, hypopharyngeal, laryngeal or tracheal strictures.

/insert Table 4 near here/

Impaired secretion management was also prevalent, with over $75 \%$ of the cohort presenting with pooling of secretions in the pharynx, suggesting a reduced awareness or ability to clear secretions. Aspiration of secretions was observed in two individuals with severe dysphagia. Two individuals who were unaware of their secretions could clear them with cued swallows. Sensation assessment revealed minimal or nil response to contact by the scope in $77 \%(\mathrm{n}=10)$ of cases. Of note, $70 \%$ of individuals with reductions in both secretion management and sensation had been identified as having burn injury to the airway on admission to hospital.

The characteristics of the swallow mechanism observed during bolus trials via FEES are displayed in table 5.The information gained from the FEES on the oral phase 
was limited to judgement on bolus delivery into the pharynx (Table 5). Over 50\% of the cohort had mild or moderate deficits with bolus delivery on both thickened and thin fluids. For those 8 individuals trialled on solids, $75 \%$ presented with mild or moderate deficits with bolus delivery.

/insert Table 5 near here/

A mild to moderate delay in swallow reflex initiation was noted in $85 \%$ of the cohort for all fluid consistencies trialled. For food textures, two participants demonstrated a mild to moderate delay in triggering the swallow reflex. Inadequate structural movements during the swallow were evident across bolus types for the entire cohort (Table 5), with up to $92 \%$ of the cohort displaying weak BOT function, inadequate pharyngeal squeeze, and decreased epiglottal inversion(i.e., reduced whiteout at the height of the swallow).

Pharyngeal residue was also a consistent characteristic, being present in at least one consistency trialled for all individuals. Distribution of residue throughout the pharynx varied considerably for each participant for each bolus type. Lodging of pharyngeal residue that remained above the airway across fluid boluses was most commonly located at the BOT (47\%), valleculae (79\%), lateral channels (74\%), pyriforms (68\%), along the pharyngeal walls (47\%) and at the posterior cricoid region (53\%). Pharyngeal residue was located on the true vocal cords and subglottic shelf in greater than $60 \%$ of individuals trialled on thin fluids. Mild-moderate pharyngeal residue was also an issue for $75 \%$ of individuals trialled on solid textures, with residue being localised to the BOT, valleculae, lateral channels and pyriforms. 
Laryngeal penetration (Penetration-Aspiration scale levels 2-5) occurred in 38\% $(n=5)$ of the cohort on thick or thin fluids boluses, with aspiration (PenetrationAspiration scale levels 6-8) being identified for $54 \%(n=7)$ of patients, predominantly on thin fluid bolus trials (Table 6). Sixty percent $(n=6 / 10)$ of penetration/aspiration events were silent (participants 10,11,12,13,14, and 18) i.e., the patient had no response to the material entering the airway and made no effect to eject the material. No penetration or aspiration was observed on trials of solid textures. Aspiration most commonly occurred after the swallow (83\% of those who demonstrated aspiration on one or more fluid consistencies; $n=5$ ). Timing of aspiration after the swallow was immediate in one case, delayed due to spill-over of residue in four cases, and a combination of both immediate and delayed in one individual. The overall rating for dysphagia severity at the conclusion of FEES for each participant is detailed in table 1. For those individuals with ongoing dysphagia, $38 \%(n=5)$ of participants had their dysphagia severity reduced as a result of their diet status being upgraded following instrumental assessment.

/insert Table 6 near here/

\section{Discussion}

Clinical and instrumental examination of swallowing dysfunction in an adult cohort in the acute stages post thermal burn demonstrated a combination of oral and pharyngeal deficits which are in agreement with the handful of previously reported single case studies $^{4,5,8,9}$. Although both clinical and instrumental assessments have their strengths and limitations, in this clinical population employing a combination of both clinical and instrumental evaluations of swallow function proved imperative to accurately 
identify the multifactorial deficits contributing to aspiration risk. There were a level of disagreement observed between the CSE and FEES findings, with CSE results failing to accurately discern the true severity of the dysphagia in all cases. Specifically, $32 \%(\mathrm{n}=$ 6/19) diagnosed as dysphagia per CSE had no dysphagia identified on FEES, and 58\% (n $=11 / 19$ ) of patients were incorrectly rated as having a more severe dysphagia on CSE than on FEES. Consequently, without instrumental evaluation, some patients would have potentially continued to receive modified texture and consistency diets unnecessarily, continuing the financial burden associated with ongoing need for altered diets and negatively impacting on return to normal oral intake for the patient. However, whilst FEES was able to identify those pharyngeal stage deficits contributing to aspiration risk, it was unable to discern oral stage deficits that may limit overall ability for oral intake. As videofluoroscopy is generally unsuitable for use in the acute burns population ${ }^{11}$, the oral stage assessment conducted as part of the CSE proved a valuable part of the total assessment process in determining oromotor status and functioning during the oral phase of the swallow in this population. Considering the presence of oral and pharyngeal stage deficits in this clinical group, information obtained from both clinical and instrumental assessment results should be reviewed in combination in order to provide a comprehensive picture of all swallowing issues.

Oromotor dysfunction in terms of weakness, while prevalent, was largely unobtrusive to swallow function in the majority of participants at the time of FEES in the current study. Long periods of muscle disuse brought on by extended intubation/ventilation durations in this population may be an attributing factor to weakness $^{29}$. Furthermore, edema of the orofacial structures for those with facial burns 
and large body burns (>20\% TBSA) may have hindered oromotor functioning within the first week post injury. These factors coupled with pain from unhealed burns may impact upon strength and range of oromotor movement willingly demonstrated by individuals during CSE, rendering the movements to be rated as suboptimal yet functional for oral intake as seen in the current study. Difficulties with the oral phase of the swallow at the time of FEES manifested solely in individuals with orofacial scarring and contractures, thus indicating that oral phase deficits may not persist past the time required for healing to occur for superficial and superficial partial thickness burns and may be limited to those with deep partial and full thickness facial burns that require surgical intervention and long healing times. This finding coincides with the body of research that links orofacial contractures and long-term oral dysphagia ${ }^{4,5}$. Nasopharyngeal, hypopharyngeal, laryngeal or tracheal strictures were not present in the cohort studied and thus did not contribute to inadequate structural movements during the pharyngeal phase of the swallow. It is feasible to hypothesise that laryngeal anchoring may occur in patients with contractures on the anterior neck, however this was not observed in this cohort. Strictures after thermal burn seem relatively uncommon and reports are sparse within the literature ${ }^{30}$, yet are well documented after chemical ingestion injuries ${ }^{31,32}$.

Numerous pharyngeal stage deficits have been found to be closely associated with increased risk of aspiration in other dysphagic populations, including pooling of saliva ${ }^{33}$, laryngotracheal pathology ${ }^{34-38}$, delayed swallow trigger ${ }^{39}$, impaired sensation ${ }^{40}$, reduced airway protection $^{41,42}$, and the presence of pharyngeal residue $e^{39,43}$. In the current study all of these features were found to be prevalent, indicating that this is a clinical cohort at risk of aspiration due to multifactorial causes. Even across the spectrum of dysphagia 
severity, participants in the current group presented with multiple factors contributing to aspiration risk. In addition, the potential for aspiration to lead to pulmonary compromise in this population was found to be further compounded by the presence of poor cough strength and advanced tooth decay. Most participants had weakened cough strength indicating the potential inability to successfully clear aspirated secretions. Furthermore the high presence of poor dentition is significant, considering the known relationship between the number of decayed teeth, missing teeth and poorly fitted dentures and an increased incidence of aspiration pneumonia ${ }^{44-49}$. Poor oral hygiene creates a hospitable environment for growth of pathogenic organisms in the mouth which further predisposes the patient to pneumonia following the aspiration of contaminated oral secretions ${ }^{49-52}$. Hence, there are a number of co-existing factors placing this clinical population at high risk for aspiration and possibly the development of aspiration pneumonia. For this reason, it is critical that a comprehensive instrumental assessment is conducted alongside the CSE to accurately identify all potential risk factors.

Decreased laryngopharyngeal sensation was observed in $77 \%$ of the current cohort and was potentially contributed to by prolonged periods of ETT intubation and ventilation and, in some cases, concomitant inhalation injury. Predisposition for poor sensation in the burn population has lead to the prediction of greater incidence of silent aspiration $^{11}$, which was a concern in the current cohort in $60 \%$ of participants who had penetration/aspiration verified during FEES. The potential for silent aspiration and its clinical implications further compounds the need for instrumental assessment of swallow function post thermal burn injury to detect physiological dysfunction that cannot be 
reliably determined via CSE. FEES is particularly suited to the burn population and may be the preferred procedure during the acute phase following burn injury due to its ability to assess sensory impairment, edema, erythema and ulceration of pharyngeal and laryngeal structures caused by concomitant inhalation injury, and its ability to be carried out at bedside when mobility and positioning are compromised by pain, splints and contractures $^{11}$. Furthermore, FEES is repeatable, which caters for the long recovery process for burn patients and allows for assessment of vocal cord integrity and function, which can be affected by endotracheal intubation and inhalation injury ${ }^{53}$.

Laryngotracheal pathology was a prevalent feature across the cohort studied, with over a large proportion of cohort demonstrating some degree of anatomical alteration in the hypopharynx and larynx. This high incidence of structural abnormality is comparable to the findings of Clayton and colleagues ${ }^{10}$ who conducted a retrospective study of patients with severe burns who required tracheostomy, and found laryngotracheal abnormalities in $100 \%$ of participants. In light of their data, Clayton et al ${ }^{10}$ proposed that the actual incidence of laryngotracheal abnormality in this population is currently underestimated. The current data would concur, and lends evidence to support that laryngotracheal pathology is a highly prevalent feature in this clinical population. It is postulated that such pathological changes may be caused by the often long periods of intubation by either an endotracheal tube or tracheostomy tube, and/or as a consequence of mucosal damage caused by inhalation injury ${ }^{54-57}$. In the current cohort, such possible causal factors were common with $>50 \%$ experiencing inhalation injury, $85 \%$ undergoing prolonged durations of intubation (i.e., 5 or more days with ETT), and $46 \%$ requiring a 
tracheostomy tube. A high incidence of laryngeal pathology within the cohort is an important finding, as it could conceivably contribute to the observed aspiration risk.

Pharyngeal edema was a significant issue for the majority of the cohort and its presence has the potential to disrupt sensation and efficiency of the swallow. Widespread edema (i.e., beyond the location of the burn injury) is an acute physiological response to burns larger than $30 \%{ }^{58}$ and is considered an early reaction which resolves within three to four days post injury in most cases ${ }^{58,59}$. Pharyngeal edema that persists past the initial injury phase, once fluid resuscitation has been achieved, may be contributed to by the presence of long term NGT placement, necessary for the management of the hypermetabolic response post burn injury. Further understanding of the impact of widespread edema on swallowing and its patterns of resolution is needed. A prospective study using repeated FEES assessment would provide valuable insight into this issue.

Aspiration of saliva is a common feature in patients that are ventilator or tracheostomy dependent ${ }^{29,37,60,61}$, and the presence of pooled saliva is reported to be associated with greater likelihood of aspiration of fluids ${ }^{33}$. It has previously been postulated that increased salivary secretions may be precipitated in this population by concomitant inhalation injury, with poor cilia functioning and sloughing of and damage to the mucosa causing increased secretion production and decreased sensation ${ }^{11,56,62}$. Although a large proportion of the cohort (70\%) displayed poor ability to manage secretions in combination with poor sensation and inhalation injury, this study fails to provide statistical evidence to support this theory. Investigation via FEES at earlier and multiple intervals post injury may provide a different insight into the effects of inhalation injury on secretion management. 
Premature spillage (mild to moderate deficits in delivery of the bolus into the pharynx), a parameter related to the presence of lingual dysfunction ${ }^{63}$ was observed in the current cohort. Although all participants were rated on the CSE as having tongue function within normal limits, it is possible that some mild degree of generalised oromotor weakness coupled with reduction in sensation may account for poor bolus containment prior to swallow initiation in this population. Furthermore, a delayed swallow trigger was a frequent deficit. Delayed reflex initiation has been recognised as being strongly associated with increased risk of aspiration ${ }^{42}$, with risk of aspiration increasing proportionally to the time the bolus dwells in the pharynx before the initiation of the swallow $^{64}$. As no participants in the current cohort were identified as having any neurological deficits, it is likely that failure to trigger a swallow promptly is solely attributable to the fact that the participant is unaware of the position of the bolus in the pharynx. While this may increase the possibility of aspiration prior to the swallow, this was not evident within the current cohort.

Specific pharyngeal phase deficits that have been reported in literature to date for patients with burn injury include decreased BOT to PPW movement, reduced pharyngeal clearance and suboptimal hyolaryngeal movement ${ }^{4,9,13}$. Similar characteristics were observed across the cohort; a large proportion presented with reduced BOT to PPW contact and abnormal pharyngeal longitudinal and constrictor movement. This may lead to a build up of residue outside the larynx, contributing to increased aspiration risk. Distribution of residue was vastly different for each participant; acknowledgement of residue location is imperative in determining the point of breakdown in the process of bolus clearance ${ }^{26}$. Residue was widely dispersed throughout the velopharynx, 
hypopharynx and larynx, suggesting either a delayed movement or reduced force in one laryngeal structure or all muscles involved in performing a swallow ${ }^{26}$. The presence of pharyngeal residue was observed to lead to aspiration after the swallow as the result of spill-over into and through the laryngeal inlet for $83 \%$ of individuals who aspirated during the assessment. Langmore ${ }^{26}$ noted that the overall severity of residue relates directly to the sensitivity/response of the patient to the existence of the residue. In the burn population altered sensation was a notable feature with $77 \%$ of patients registering no response to light touch from the scope on assessment.

The current data would suggest that the deficits contributing to dysphagia post burn relate primarily to disruption to the structure and function of the swallowing mechanism, largely due to edema and orofacial contracture formation. This information has implications for rehabilitation, as it appears the swallow does not necessarily need to be retrained at a neurological level, but requires compensatory management of oral and pharyngeal deficits through postural adjustment, training of airway protection strategies and the use of texture and consistency modification to minimise aspiration risk coupled with early and ongoing intervention to minimise/prevent any oral contractures ${ }^{5,65,66}$. Due to the medically fragile nature of this population and the fact that recovery patterns for the physiological deficits identified by the current study, such as edema, is unknown, regular monitoring of swallow function via both CSE and FEES is recommended to optimise the rehabilitation process. 


\section{Conclusion}

Currently there is very little literature investigating the nature of the swallowing impairment post thermal burn injury. Findings of this study identified generalised oromotor weakness was observed upon CSE in the majority of the cohort, with functional deficits of the oral phase reserved for the few individuals with severe dysphagia and orofacial contractures. Observable physiological deficits on FEES that were identified as being prominent across the cohort included: laryngotracheal pathology, decreased ability to manage secretions, delayed swallow initiation, decreased laryngopharyngeal sensation, diffuse pharyngeal residue and a risk of silent aspiration. The need for the introduction and routine utilisation of instrumental assessment of swallowing function in some cases post thermal burn injury has been highlighted due to the multifaceted nature for the dysphagia and the possibility for silent aspiration, a feature undetectable using clinical beside evaluation of swallowing. This study has provided a first detailed report of the physiological effects of burn injury on swallow function in a group of patients with varying degrees of dysphagia. The data presented in this paper facilitates a greater understanding of the physiological underpinnings of dysphagia in the burn population, as well as promotes the inclusion of SLP services and the use of instrumental evaluations of swallowing with this population. Further research however is needed through systematic routine FEES assessment of a large prospective cohort in order to examine physiological changes and recovery over time. 


\section{Acknowledgements}

The authors gratefully acknowledge funding support for this study from the Royal

Brisbane and Women's Hospital Foundation. We acknowledge the assistance of the Royal Brisbane and Women's Hospital Professor Stuart Pegg Adult Burns Unit in the recruitment of participants for this study. Special thanks to the Royal Brisbane and Women's Hospital ENT department for their assistance with the assessments. Finally, the authors wish to acknowledge the 19 individuals that participated in this study for the generous gift of their time, patience and courage. 


\section{References}

1. Rumbach AF, Ward EC, Cornwell PL, Bassett LV, Khan A, Muller MJ:

Incidence and predictive factors for dysphagia following thermal burn injury: A prospective cohort study. J Burn Care Res, in press.

2. Edelman DA, Sheehy-Deardorff DA, White MT: Bedside assessment of swallowing is predictive of an abnormal barium swallow examination. J Burn Care Res 29: 89-96, 2008.

3. McKinnon DuBose C, Groher MG, Carnaby Mann GS, Mozingo DW:Pattern of dysphagia recovery after thermal burn injury. J Burn Care Rehabil 26: 233-237, 2005.

4. Rumbach AF, Ward EC, Cornwell PL, Bassett LV, Muller MJ: The challenges of dysphagia management and rehabilitation after extensive thermal burn injury: A complex case. J Burn Care Res 30: 899-903, 2009.

5. Rumbach AF, Ward EC, Cornwell PL, Bassett LV, Spermon ML, Plaza AL, Muller MJ: Dysphagia management and rehabilitation: an interdisciplinary and multidisciplinary collaborative. J Med Speech Lang Pathol 19: 25-34, 2011.

6. Rumbach AF, Ward EC, Cornwell PL, Bassett LV, Muller MJ: Clinical progression and outcome of dysphagia following thermal burn injury: a prospective cohort study, J Burn Care Res, in press.

7. Ward EC, Uriarte M, Conroy A-L: Duration of dysphagic symptoms and swallowing outcomes after thermal burn injury. J Burn Care Rehabil 22:441-453, 2001. 
8. Clayton NA, Kennedy PJ: Management of firecracker induced oropharyngeal burns: a case report. Advances in Speech-Language Pathology 9: 265-270, 2007.

9. Clayton NA, Ledgard JP, Haertsch PA, Kennedy P, Maitz P: Rehabilitation of speech and swallowing after burns reconstructive surgery of the lips and nose. $\mathrm{J}$ Burn Care Res 30: 1039-1045, 2009.

10. Clayton N, Kennedy P, Maitz P: The severe burns patient with tracheostomy: Implications for management of dysphagia, dysphonia and laryngotracheal pathology. Burns 36: 850-855, 2010.

11. Rumbach AF, Ward EC, McKinnon DuBose C, Clayton NA: Burn Injury. In: Ward EC, Morgan AT (eds.): Dysphagia post trauma. San Francisco: Plural Publishing, pp 151-199.

12. Wust KJ: A modified dynamic mouth splint for burn patients. J Burn Care Res 27: 86-92, 2006.

13. Muehlberger T, Kunar D, Munster A, Couch M: Efficacy of fiberoptic laryngoscopy in the diagnosis of inhalation injuries. Arch Otolaryngol Head Neck Surg 124: 1003-1007, 1998.

14. DePippo KL, Holas MA, Reding MJ: Validation for the 3-oz water swallow test for aspiration following stroke. Arch Neurol 49: 1259-1261, 1992.

15. Garon BR, Engle M, Ormiston C: Reliability of the 3-oz water swallow test utilising cough reflex as sole indicator of aspiration. Neurorehabilitation and Neural Repair, 9: 139-143, 1995.

16. Suiter DM, Leder SB: Clinical utility of the 3-ounce water swallow test. Dysphagia 23: 244-250, 2008. 
17. Wu M-C, Chang Y-C, Wang T-G, Lin LC: Evaluating swallowing dysfunction using a 100-ml water swallowing test. Dysphagia 19: 43-47, 2004.

18. Langmore SE, Schatz K, Olsen N: Fiberoptic endoscopic examination of swallowing safety: a new procedure. Dysphagia 2: 216-219, 1988.

19. Dieticians Association of Australian and the Speech Pathology Association of Australia Limited: Texture-modified foods and thickened fluids as used for individuals with dysphagia: Australian standardised labels and definitions. Nutrition and Dietetics 64: S53-S76, 2007.

20. Adnerhill I, Ekberg O, Groher ME: Determining normal bolus size for thin liquids. Dysphagia 4: 1-3, 1989.

21. Kelly AM, Leslie P, Beale T, Payten C, Drinnan MJ: Fiberopic endoscopic evaluation of swallowing and videofluoroscopy: does examination type influence perception of pharyngeal residue severity?. Clin Otolaryngol 31: 425-432, 2006.

22. McGowan SL, Gleeson M, Smith M, Hirsch N, Shuldham CM: A pilot study of fiberoptic endsoscopic evaluation of swallowing in patients with cuffed tracheostomies in neurological intensive care. Neurocritical Care 6:90-93, 2007.

23. Robbins J, Levine R, Maser A, Rosenbek JC, Kempster GB: Swallowing after unilateral stroke of the cerebral cortex. Arch Phys Med Rehabil 74: 1295-1300, 1993.

24. Tracy JF, Logemann JA, Kahrilas PJ, Jacob P, Kobara M, Krugler C: Preliminary observations on the effects of age on oropharyngeal deglutition. Dysphagia 4: 9094, 1989. 
25. Landis JR, Koch GG:The measurement of observer agreement for categorical data. Psychol Bull 86: 420-428, 1979.

26. Langmore SE: Fiberoptic evaluation and treatment of swallowing disorders. New York: Thieme, 2001.

27. Donzelli J, Brady S, Wesling M, Craney M: Predictive value of accumulated oropharyngeal secretions for aspiration during video nasal endoscopic evaluation of the swallow. Ann Otol Rhinol Laryngol 112:469-475, 203.

28. Rosenbek JC, Robbins JA, Roecker EB, Coyle JL, Wood JL: A PenetrationAspiration Scale. Dysphagia 11:93-98, 1996.

29. DeVita MA, Spierer-Runback L: Swallowing disorders in patients with prolonged orotracheal intubation or tracheostomy tubes. Crit Care Med 18:1328-1330, 1990.

30. Gaissert HA, Lofgren RH, Grillo HC: Upper airway compromise after inhalation injury: Complex strictures of the larynx and trachea and their management. Annals of Surgery 218:672-678, 1993.

31. Hoseok I, Shim YM, Son YI, Kim K, Choi YS: Pharyno-colostomy with supraglottic partial laryngectomy in caustic oropharyngeal stricture. Annals of Thoracic Surgery 81: 712-714, 2006.

32. Shikowitz MJ, Levy J, Villano D, Graver ML, Pochaczevsky P: Speech and swallowing rehabilitation following devastating caustic ingestion: Techniques and indicators for success. Laryngoscope 106: 1-12, 1996.

33. Murray J, Langmore SE, Ginsberg S, Dostie A: The significance of accumulated oropharyngeal secretions and swallowing frequency in predicting aspiration.

Dysphagia 11: 99-103, 1996. 
34. Colice GL: Resolution of laryngeal injury following translaryngeal intubation. Am Rev Respir Dis 145: 361-364, 1992.

35. Colice GL, Stukel TA, Dain B: Laryngeal complications of prolonged intubation. Chest 96: 877-884, 1989.

36. Stauffer JL, Olson DE, Petty TL: Complications and consequences of endotracheal intubation and tracheotomy. Am J Med 10: 65-76, 1981.

37. Tolep K, Getch CL, Criner GJ: Swallowing dysfunction in patients receiving prolonged mechanical ventilation. Chest 109:167-172, 1996.

38. Whited RE: A prospective study of laryngotracheal sequelae in long-term intubation. Laryngoscope 94: 367-377, 1984.

39. Perlman AL, Booth BM, Grayhack JP: Videofluoroscopic predictors of aspiration in patients with oropharyngeal dysphagia. Dysphagia 9: 90-95, 1994.

40. Aviv JE, Kim T, Goodhart K, Kaplan S, Thomson J, Diamond B, Close LG: FEESST: a new bedside endoscopic test of the motor and sensory components of swallowing. Ann Otol Rhinol Laryngol 107: 378-387, 1998.

41. Kahrilas PJ, Lin S, Rademaker AW, Logemann JA: Impaired deglutitive airway protection: a videofluoroscopic analysis of severity and mechanism. Gasteroenterology, 113: 1457-1464, 1997.

42. Lundy DS, Smith C, Colangelo L, Sullivan PA, Logemann JA, Lazarus CL, Newman LA, Murry T, Lombard L, Gaziano J: Aspiration: cause and implications. Otolaryngol Head Neck Surg 120: 474-478, 1999.

43. Eisenhuber E, Schima W, Schober E, Pokieser P, Stadler A, Scharitzer M, Oschatz E: Videofluroscopic assessment of patients with dysphagia: pharyngeal 
retention is a predictive factor for aspiration. Am J Roentgenol 178: 393-398, 2002.

44. Bartlett JG, Finegold SM. Anaerobic infections of the lung and pleural space. Am Rev Respir Dis 110: 56-77, 1974.

45. Langmore SE, Terpenning MS, Schork A, Chen Y, Murray JD, Lopatin D, Losche WJ. Predictors of aspiration pneumonia: How important is dysphagia? Dysphagia 13:69-81, 1998.

46. Mojon P, Budtz-Jorgensen E, Michel JP et al. Oral health and history of respiratory tract infection in frail institutionalized elders. Gerodontology 14:9-16, 1997.

47. Mojon P, Rentsch A, Budtz-Jorgensen E et al. Effects of an oral health program on selected clinical parameters and salivary bacteria in a long-term care facility. Eur J Oral Sci 106: 827-834, 1998.

48. Scannapieco FA, Papandontos GD, Dunford RG. Associations between oral conditions and respiratory disease in a national sample survey population. Ann Periodontol 3: 251-256, 1998.

49. Terpenning MS, Taylor GW, Lopatin DE, Kinder C, Dominguez L, Loesche WJ. Aspiration pneumonia: Dental and oral risk factors in an older veteran population. J Am Geriat Soc 49: 557-563, 2001.

50. Abe S, Ishihara K, Adachi M, Okuda K. Oral hygiene evaluation for effective oral care in preventing pneumonia in dentate elderly. Arch Gerontol Geriatr 43: 53-64, 2006. 
51. Bartlett JG, Gorbach SL, Finegold SM. The bacteriology of aspiration pneumonia. Am J Med 56: 202-207, 1974.

52. Yoneyama T, Yoshida M, Ohrui T, Mukaiyama H, Okamoto H, Hoshiba K, et al. Oral care reduces pneumonia in older patients in nursing homes. J Am Geriatr Soc 50: 430-434, 2002.

53. Leder SB, Sasaki CT, Burrell MI: Fiberoptic endoscopic evaluation of dysphagia to identify silent aspiration. Dysphagia 18: 19-21, 1998.

54. Clement P, Hans S, de Mones E, Sigston E, Laccourreye O, Brasnu D: Dilatation for assisted ventilation-induced laryngotracheal stenosis. Laryngoscope 115: 1595-1598, 2005.

55. Flexon PB, Cheney ML, Montgomery WW, Turner PA: Management of patients with glottic and subglottic stenosis resulting from thermal burns. Ann Otol Rhinol Laryngol 98: 27-30, 1989.

56. Gaissert HA, Lofgren RH, Grillo HC: Upper airway compromise after inhalation injury: Complex strictures of the larynx and trachea and their management. Ann Surg 218: 672-678, 1993.

57. Goldsmith T: Evaluation and treatment of swallowing disorders following endotracheal intubation and tracheostomy. Int Anesthesiol Clin 38: 219-242, 2000.

58. Cook D: Pathophysiology of burns. In: Bosworth Bousfield C (ed): Burn trauma: Management and nursing care. London: Whurr, pp 1-12.

59. Pankhurst S, Pochkhanawala T: Wound Care. In: Bosworth Bousfield C (ed): Burn trauma: Management and nursing care. London: Whurr, pp 81-108.. 
60. Elpern EH, Scott MG, Petro L, Ries MH: Pulmonary aspiration in mechanically ventilated patients with tracheostomies. Chest 105: 563-566, 1994.

61. Leder SB: Incidence and type of aspiration in acute care patients requiring mechanical ventilation via a new tracheostomy. Chest 122: 1721-1726, 2002.

62. Cohen MA, Guzzardi LJ: Inhalation of products of combustion. Ann Emerg Med 12: 628-632, 1983.

63. Logemann JA: Manual for the videofluorographic study of swallowing. Austion TX: Pro-Ed, 1993.

64. Arvedson JC, Lefton-Greif MA: Pediatric videofluoroscopic swallow studies. San Antonio TX: Communication Skill Builders, 1998.

65. Australian and New Zealand Burn Association: Report: Burns Workforce Survey 2007 (Allied Health). Brisbane, Australia, in preparation.

66. Clayton N, Whitney S, O’Loughlin G, Crouch A, Patterson M: Speech Pathology. In D. Edgar (Ed.), Burn survivor rehabilitation: Principles and guidelines for the allied health professional (pp. 74-88). Brisbane, Australia: Australian and New Zealand Burn Association, 2005. 
Table 1 Biographical information for 19 participants who underwent FEES assessment for investigation of dysphagia post thermal burn injury

\begin{tabular}{|c|c|c|c|c|c|c|c|c|c|c|c|c|c|}
\hline $\begin{array}{c}\text { Participant } \\
\text { number }\end{array}$ & Gender & Age & $\begin{array}{c}\text { Burn } \\
\text { Etiology }\end{array}$ & $\begin{array}{c}\% \\
\text { TBSA }\end{array}$ & $\begin{array}{c}\text { Inhalation } \\
\text { injury }\end{array}$ & $\begin{array}{l}\text { Facial } \\
\text { burns }\end{array}$ & $\begin{array}{l}\text { Duration } \\
\text { of ETT } \\
\text { (days) }\end{array}$ & $\begin{array}{l}\text { Ventilation } \\
\text { duration } \\
\text { (days) }\end{array}$ & $\begin{array}{l}\text { Tracheostomy } \\
\text { In Situ during } \\
\text { admission }\end{array}$ & $\begin{array}{c}\text { Initial } \\
\text { referral to } \\
\text { SLP (days } \\
\text { from } \\
\text { admission) }\end{array}$ & $\begin{array}{c}\text { Duration } \\
\text { to FEES } \\
\text { (days from } \\
\text { admission) }\end{array}$ & $\begin{array}{l}\text { Dysphagia } \\
\text { severity } \\
\text { Pre FEES }\end{array}$ & $\begin{array}{l}\text { Dysphagia } \\
\text { severity }^{\#}- \\
\text { Post FEES }\end{array}$ \\
\hline 1 & $\mathrm{M}$ & 54 & Flame & 20 & $\mathrm{Y}$ & $\mathrm{Y}^{\infty}$ & 21 & 21 & $\mathrm{~N}$ & 24 & 38 & Severe & Moderate $^{\sim}$ \\
\hline 2 & $\mathrm{~F}$ & 35 & Flame & 20 & $\mathrm{~N}$ & $\mathrm{~N}$ & 13 & 13 & $\mathrm{~N}$ & 1 & 41 & Moderate & Resolved \\
\hline 3 & $\mathrm{M}$ & 58 & Flash & 4 & $\mathrm{Y}$ & $\mathrm{Y}$ & 5 & 5 & $\mathrm{~N}$ & 6 & 12 & Mild & Resolved \\
\hline 4 & $\mathrm{~F}$ & 39 & Flame & 37 & $\mathrm{~N}$ & $\mathrm{Y}$ & 0 & 0 & $\mathrm{~N}$ & 1 & 5 & Mild & Resolved \\
\hline 5 & $\mathrm{M}$ & 60 & Flame & 53.5 & $\mathrm{Y}$ & $Y^{\infty}$ & 13 & 41 & $\mathrm{Y}^{\wedge}$ & 44 & 80 & Severe & Severe $^{\sim}$ \\
\hline 6 & $\mathrm{M}$ & 27 & Flame & 29 & $\mathrm{Y}$ & $\mathrm{Y}$ & 15 & 15 & $\mathrm{~N}$ & 16 & 29 & Moderate & Resolved \\
\hline 7 & $\mathrm{M}$ & 38 & Flame & 18 & $\mathrm{Y}$ & $\mathrm{Y}$ & 5 & 5 & $\mathrm{~N}$ & 6 & 14 & Moderate & Moderate $^{\sim}$ \\
\hline 8 & $\mathrm{~F}$ & 18 & Scald & 22 & $\mathrm{~N}$ & $\mathrm{Y}$ & 15 & 15 & $\mathrm{~N}$ & 4 & 30 & Severe & Moderate $^{\sim}$ \\
\hline 9 & M & 54 & Combination & 24.5 & $\mathrm{Y}$ & $\mathrm{Y}$ & 17 & 17 & $\mathrm{~N}$ & 19 & 31 & Severe & Moderate $^{\sim}$ \\
\hline 10 & $\mathrm{~F}$ & 31 & Scald & 40 & $\mathrm{~N}$ & $\mathrm{~N}$ & 5 & $5 *$ & $\mathrm{Y}^{\wedge}$ & 6 & 59 & Severe & Severe $^{\sim}$ \\
\hline 11 & $\mathrm{M}$ & 43 & Flame & 51 & $\mathrm{Y}$ & $\mathrm{Y}$ & 24 & 41 & $\mathrm{Y}$ & 45 & 66 & Moderate & Moderate $^{\sim}$ \\
\hline 12 & $\mathrm{M}$ & 64 & Flame & 39 & $\mathrm{~N}$ & $\mathrm{Y}$ & 23 & 36 & $\mathrm{Y}^{\wedge}$ & 38 & 65 & Severe & Severe $^{\sim}$ \\
\hline 13 & $\mathrm{M}$ & 64 & Flame & 20 & $\mathrm{~N}$ & $\mathrm{~N}$ & 0 & 0 & $\mathrm{~N}$ & 1 & 12 & Severe & Severe $^{\sim}$ \\
\hline 14 & $\mathrm{M}$ & 80 & Flame & 30 & $\mathrm{~N}$ & $\mathrm{~N}$ & 42 & 3 & $\mathrm{~N}$ & 9 & 25 & Severe & Severe $^{\sim}$ \\
\hline 15 & $\mathrm{M}$ & 85 & Combination & 11 & $\mathrm{Y}$ & $\mathrm{Y}$ & 12 & 12 & $\mathrm{~N}$ & 13 & 24 & Moderate & Resolved \\
\hline 16 & $\mathrm{M}$ & 30 & Flame & 66.5 & $\mathrm{Y}$ & $\mathrm{Y}$ & 20 & 36 & $\mathrm{Y}^{\wedge}$ & 36 & 64 & Severe & Moderate $^{\sim}$ \\
\hline 17 & $\mathrm{M}$ & 18 & Flame & 63 & $\mathrm{Y}$ & $\mathrm{Y}$ & 4 & 17 & $\mathrm{Y}$ & 17 & 54 & Mild & Resolved \\
\hline 18 & $\mathrm{~F}$ & 32 & Flame & 22 & $\mathrm{Y}$ & $\mathrm{Y}$ & 13 & 13 & $\mathrm{~N}$ & 14 & 18 & Severe & Severe $^{\sim}$ \\
\hline 19 & $\mathrm{M}$ & 81 & Combination & 35 & $\mathrm{~N}$ & $\mathrm{~N}$ & 1 & 1 & $\mathrm{~N}$ & 3 & 27 & Moderate & Mild \\
\hline
\end{tabular}

Note: $\mathrm{M}=$ Male, $\mathrm{F}=$ Female, $\mathrm{ETT}=$ endotracheal intubation, $\mathrm{SLP}=$ speech-language pathology, $\mathrm{TBSA}=$ total body surface area, $\mathrm{Y}=$ yes, $\mathrm{N}=$ no, $\infty$ orofacial scarring and contractures present $\mathcal{N}$ Ventilatory support provided at initial hospital admission only; * additional period of ventilatory support required during inpatient stay $\wedge$ tracheostomy tube in situ at time of FEES; ${ }^{*}$ Dysphagia severity as per purpose-built scale for the burn population based on levels of diet restriction only; $~$ penetration observed; $\sim$ aspiration observed. 
Table 2 Interrater reliability using weighted kappa statistics

\begin{tabular}{|c|c|c|c|c|c|c|}
\hline Erythema & 88.16 & 84.42 & 0.2400 & 0.1598 & 1.50 & 0.0666 \\
\hline Marionjoy Secretion rating scale & 99.42 & 79.66 & 0.9713 & 0.2290 & 4.24 & 0.0000 \\
\hline Reflex initiation & 96.05 & 82.06 & 0.7799 & 0.2126 & 3.67 & 0.0001 \\
\hline Inadequacy of structural movements during swallow & 98.47 & 52.80 & 0.9677 & 0.0874 & 11.08 & 0.0000 \\
\hline Pharyngeal Residue & 98.83 & 80.36 & 0.9404 & 0.2290 & 4.11 & 0.0000 \\
\hline
\end{tabular}


Table 3 Intrarater reliability using weighted kappa statistics (based on 5 repeat viewings per rater)

\begin{tabular}{|c|c|c|c|c|c|c|c|}
\hline & Parameter & Agreement (\%) & $\begin{array}{l}\text { Expected } \\
\text { Agreement (\%) }\end{array}$ & Kappa & Standard Error & $\mathrm{Z}$ & Prob $>Z$ \\
\hline \multirow[t]{8}{*}{ Rater A } & Edema & 95 & 85 & 0.6667 & 0.3771 & 1.77 & 0.0385 \\
\hline & Erythema & 100 & 68 & 1.00 & 0.4472 & 2.24 & 0.0127 \\
\hline & Marionjoy Secretion rating scale & 100 & 80 & 1.00 & 0.4472 & 2.24 & 0.0127 \\
\hline & Delivery of Bolus into Pharynx & 80 & 56 & 0.5455 & 0.3983 & 1.37 & 0.0855 \\
\hline & Reflex Initiation & 100 & 52 & 1.00 & 0.4472 & 2.24 & 0.0127 \\
\hline & Inadequacy of structural movements during swallow & 100 & 80 & 1.00 & 0.4472 & 2.24 & 0.0127 \\
\hline & Pharyngeal Residue & 94.29 & 51.02 & 0.8833 & 0.1690 & 5.23 & 0.0000 \\
\hline & Penetration-Aspiration Scale & 100 & 72.22 & 1.00 & 0.3536 & 2.83 & 0.0023 \\
\hline \multirow[t]{8}{*}{ Rater B } & Edema & 90 & 80 & 0.5 & 0.3464 & 1.44 & 0.0745 \\
\hline & Erythema & 60 & 52 & 0.1667 & 0.4472 & 0.37 & 0.3547 \\
\hline & Marionjoy Secretion rating scale & 95 & 75 & 0.800 & 0.422 & 1.89 & 0.0294 \\
\hline & Delivery of Bolus into Pharynx & 95 & 85 & 0.6667 & 0.3771 & 1.77 & 0.0385 \\
\hline & Reflex Initiation & 95 & 77 & 0.7826 & 0.3810 & 2.05 & 0.0200 \\
\hline & Inadequacy of structural movements during swallow & 77.14 & 50.04 & 0.5425 & 0.1690 & 3.21 & 0.0007 \\
\hline & Pharyngeal Residue & 95.56 & 82.22 & 0.7500 & 0.3606 & 2.08 & 0.0188 \\
\hline & Penetration-Aspiration Scale & 96.09 & 75.39 & 0.8413 & 0.3498 & 2.41 & 0.0081 \\
\hline
\end{tabular}


Table 4 Anatomic-Physiologic data collected during FEES for 13 individuals post burn

\begin{tabular}{|l|c|}
\hline \multicolumn{1}{|c|}{ Parameter } & $\begin{array}{c}\text { Presence of Characteristic } \\
\text { N (\%) }\end{array}$ \\
\hline Reduced velopharyngeal closure & $2(15)$ \\
\hline Reduced BOT and pharyngeal muscle movement & $8(62)$ \\
\hline Altered structural appearance of the hypopharynx and larynx & $5(38)$ \\
Laryngeal granuloma (unilateral or bilateral) & $3(23)$ \\
Laryngeal ulceration (unilateral or bilateral) & $10(77)$ \\
Edema & $5(38)$ \\
Erythema & \\
\hline Altered laryngeal function & $7(54)$ \\
Decreased TVC movement (unilateral or bilateral) & $7(54)$ \\
Glottic gap on phonation & $4(31)$ \\
FVC/AP constriction & $3(23)$ \\
\hline Secretion management: Marionjoy secretion rating scale & $6(46)$ \\
Normal & $0(0)$ \\
Mild & $2(15)$ \\
Moderate & $2(15)$ \\
Severe & \\
Profound & \\
\hline
\end{tabular}

Note: $\mathrm{AP}=$ anterior-posterior; $\mathrm{BOT}=$ base of tongue $; \mathrm{FVC}=$ false vocal cord $; \mathrm{TVC}=$ true vocal cord 
Table 5 Oral and pharyngeal phase swallowing characteristics observed during FEES for 13 individuals post thermal burn

\begin{tabular}{|c|c|c|c|}
\hline \multirow[t]{3}{*}{ Parameter } & \multicolumn{3}{|c|}{ Rating/Presence of characteristic per bolus type } \\
\hline & \multicolumn{3}{|c|}{$\mathrm{N}(\%)$} \\
\hline & Thick fluids & Thin fluids & Solids \\
\hline \multicolumn{4}{|c|}{ Oral Phase } \\
\hline $\begin{array}{l}\text { Delivery of bolus into pharynx } \\
\text { Normal } \\
\text { Mild } \\
\text { Moderate } \\
\text { N/A }\end{array}$ & $\begin{array}{c}2(15) \\
8(62) \\
3(23) \\
0(0)\end{array}$ & $\begin{array}{l}2(15) \\
5(38) \\
3(23) \\
3(23)\end{array}$ & $\begin{array}{c}2(15) \\
5(38) \\
1(8) \\
5(38)\end{array}$ \\
\hline \multicolumn{4}{|c|}{ Pharyngeal Phase } \\
\hline $\begin{array}{l}\text { Reflex Initiation } \\
\text { Normal } \\
\text { Mild } \\
\text { Moderate } \\
\text { N/A }\end{array}$ & $\begin{array}{c}6(46) \\
5(38) \\
2(15) \\
0(0)\end{array}$ & $\begin{array}{c}5(38) \\
4(31) \\
1(8) \\
3(23)\end{array}$ & $\begin{array}{c}6(46) \\
1(8) \\
1(8) \\
5(38)\end{array}$ \\
\hline $\begin{array}{l}\text { Inadequacy of structural movements during swallow } \\
\text { BOT } \\
\text { Pharyngeal constrictors } \\
\text { Epiglottal inversion } \\
\text { Velar elevation } \\
\text { Arytenoid tilt } \\
\text { TVC/FVC adduction } \\
\end{array}$ & $\begin{array}{l}12(92) \\
11(85) \\
9(69) \\
1(8) \\
1(8) \\
5(38) \\
\end{array}$ & $\begin{array}{l}8(62) \\
8(62) \\
6(46) \\
1(8) \\
0(0) \\
3(23) \\
\end{array}$ & $\begin{array}{l}6(46) \\
6(46) \\
4(31) \\
1(8) \\
0(0) \\
3(23) \\
\end{array}$ \\
\hline $\begin{array}{l}\text { Pharyngeal residue } \\
\text { Normal } \\
\text { Mild } \\
\text { Moderate } \\
\text { Severe } \\
\text { N/A }\end{array}$ & $\begin{array}{l}2(15) \\
7(54) \\
2(15) \\
2(15) \\
0(0)\end{array}$ & $\begin{array}{l}3(23) \\
5(38) \\
1(8) \\
1(8) \\
3(23) \\
\end{array}$ & $\begin{array}{l}2(15) \\
5(38) \\
1(8) \\
0(0) \\
5(38)\end{array}$ \\
\hline
\end{tabular}

Note: $\mathrm{BOT}=$ base of tongue $; \mathrm{FVC}=$ false vocal cord; N/A $=$ not applicable/not trialled; $\mathrm{TVC}=$ true vocal cords 
Table 6 Swallow performance for 13 individuals on fluid and solid boluses as rated on the Penetration-Aspiration scale ${ }^{27}$

\begin{tabular}{|c|c|c|c|}
\hline Penetration-Aspiration Scale & Thick fluids & Thin fluids & Solids \\
\hline 1: Material does not enter the airway & $10(77)$ & $1(8)$ & $8(62)$ \\
\hline 2: Material enters the airway, remains above the VFs and is ejected from the airway & $0(0)$ & $1(8)$ & $0(0)$ \\
\hline 3: Material enters the airway, remains above the VFs and is not ejected from the airway & $1(8)$ & $2(15)$ & $0(0)$ \\
\hline 4: Material enters the airway, contacts the VFs and is ejected from the airway & $0(0)$ & $0(0)$ & $0(0)$ \\
\hline 5: Material enters the airway, contacts the VFs and is not ejected from the airway & $1(8)$ & $1(8)$ & $0(0)$ \\
\hline 6: Material enters the airway, passes below the VFs and is ejected into the larynx or out of the airway & $0(0)$ & $0(0)$ & $0(0)$ \\
\hline 7: Material enters the airway, passes below the VFs and is not ejected from the trachea despite effort & $0(0)$ & $2(15)$ & $0(0)$ \\
\hline 8: Material enters the airway, passes below the VFs and no effort is made to eject. & $1(8)$ & $3(23)$ & $0(0)$ \\
\hline N/A: Consistency/texture not trialled & $0(0)$ & $3(23)$ & $5(38)$ \\
\hline
\end{tabular}




\section{Appendix A}

\section{Delivery of bolus into pharynx}

$1=$ Normal

- Prompt delivery of bolus; No leakage of thin fluids; Small amount of leakage for dual consistencies may occur

$2=$ Mild impairment

- Slightly delayed transfer; Incompletely masticated bolus; Small amount of leakage for thin fluids

$3=$ Moderate impairment

- Moderate amount of leakage during oral prep; Difficulty using controlled swallow; Incompletely masticated bolus; Poor bolus cohesion

$4=$ Severe impairment

- Large amount of leakage during oral prep; Minimal or no mastication of bolus; Incohesive bolus, spread diffusely; No attempt at transfer

\section{Reflex Initiation (Timing of bolus flow and initiation of swallow)}

$1=$ Normal

- Bolus dwelling at base of tongue and/or valleculae for 0-1 seconds

2 = Mild impairment for $2-5$ seconds

- Bolus dwelling at valleculae, overspilling epiglottis, lateral channels and/or pyriform sinuses

3 = Moderate impairment for 6-8 seconds

- Bolus dwelling at valleculae, overspilling epiglottis, lateral channels and/or pyriform sinuses

$4=$ Severe impairment for $>9$ seconds

- Bolus dwelling at valleculae, overspilling epiglottis, lateral channels and/or pyriform sinuses.

- Absent reflex

\section{Pharyngeal residue post swallow (Observations after the swallow)}

$1=$ Normal

- No residue; Minimal coating

$2=$ Mild impairment

- Slight residue; Diffuse coating throughout pharynx; Slight pooling in pharyngeal cavities

$3=$ Moderate impairment

- Pooling in cavities (c. up to $50 \%$ )

$4=$ Severe impairment

- Fills and/or overflows cavities; May overflow into laryngeal vestibule. 